\title{
Complications and Short-Term Outcomes of Medial Opening Wedge High Tibial Osteotomy Using a Locking Plate for Medial Osteoarthritis of the Knee
}

\author{
Seung-Suk Seo, MD, Ok-Gul Kim, MD, Jin-Hyeok Seo, MD, Do-Hoon Kim, MD, Youn-Gu Kim, MD, and \\ In-Seung Lee, $\mathrm{MD}$ \\ Department of Orthopaedics, Bumin General Hospital, Busan, Korea
}

\begin{abstract}
Purpose: The purpose of this study was to investigate complications and radiologic and clinical outcomes of medial opening wedge high tibial osteotomy (MOWHTO) using a locking plate.

Materials and Methods: This study reviewed 167 patients who were treated with MOWHTO using a locking plate from May 2012 to June 2014. Patients without complications were classified into group 1 and those with complications into group 2. Medical records, operative notes, and radiographs were retrospectively reviewed to identify complications. Clinically, Oxford Knee score and Knee Injury and Osteoarthritis Outcome score (KOOS) were evaluated.

Results: Overall, complications were observed in 49 patients (29.3\%). Minor complications included lateral cortex fracture (15.6\%), neuropathy (3.6\%), correction loss $(2.4 \%)$, hematoma (2.4\%), delayed union (2.4\%), delayed wound healing (2.4\%), postoperative stiffness (1.2\%), hardware irritation (1.2\%), tendinitis (1.2\%), and hardware failure without associated symptoms (0.6\%). Major complications included hardware failure with associated symptoms $(0.6 \%)$, deep infection $(0.6 \%)$, and nonunion $(0.6 \%)$. At the first-year follow-up, there were no significant differences in radiologic measurements between groups 1 and 2 . There were no significant differences in knee scores except for the KOOS pain score.

Conclusions: Our data showed that almost all complications of the treatment were minor and the patients recovered without any problems. Most complications did not have a significant impact on radiologic and clinical outcomes.
\end{abstract}

Keywords: Knee, Osteoarthritis, Osteotomy, Open wedge, Plate, Complication

\section{Introduction}

High tibial osteotomy (HTO) is regarded as a well-established surgical procedure for management of varus axial malalignment associated with medial compartment osteoarthritis and medi-

Received May 27, 2016; Revised August 15, 2016;

Accepted September 5, 2016

Correspondence to: Seung-Suk Seo, MD

Department of Orthopaedics, Bumin General Hospital,

59 Mandeok-daero, Buk-gu, Busan 46555, Korea

Tel: +82-51-330-3000, Fax: +82-51-337-5041

E-mail: wellknee@gmail.com

Source of funding: This research will be funded by Bumin Hospital Group of Indang Medical Foundation.

This is an Open Access article distributed under the terms of the Creative Commons Attribution Non-Commercial License (http://creativecommons.org/licenses/by-nc/4.0/) which permits unrestricted non-commercial use, distribution, and reproduction in any medium, provided the original work is properly cited. cal compartment cartilage defect. It was introduced by Jackson in 1958 and commonly used by Conventry. The goal of HTO is to transfer the weight bearing load to the undamaged lateral compartment. It can delay the need for total knee arthroplasty. In particular, younger patients and active older patients $(<60$ years old) can benefit from the procedure ${ }^{1)}$.

The traditional procedure is the lateral closing wedge HTO, but it has several disadvantages: risk of peroneal nerve palsy, difficulty of acquiring a target correction, and resection of the fibula. For these reasons, medial opening wedge HTO (MOWHTO) has become a more common procedure ${ }^{2,3)}$. MOWHTO has achieved remarkable improvement not only in surgical techniques but also in surgical instruments. In recent years, locking plates that are relatively resistant to torsion and compression loads and facilitate early ambulation were developed. Several studies have reported excellent results of MOWHTO with locking plates ${ }^{4,5)}$, whereas there have been some reports on complications of the 
procedure ${ }^{3,6,7)}$. However, these reports involved a small number of subjects and lacked evaluation on the influence of such complications on clinical outcomes.

The purpose of the present study was to investigate the complications of MOWHTO using a locking plate performed at our institution. Additionally we aimed to compare radiologic outcomes and patient-reported clinical outcomes between the complicated group and the uncomplicated group.

\section{Materials and Methods}

Institutional Review Board approval was obtained. Patients who underwent MOWHTO using the TomoFix plate (DePuy Synthesis, West Chester, PA, USA) from May 2012 to June 2014 were included in this study. Patients who underwent MOWHTO with chondral resurfacing or concomitant cruciate ligament surgery were excluded. There were a total of 167 patients (43 males and 127 females) with a mean age of $55.6 \pm 8.07$ years. In the first year after surgery, patient's medical records, operation records, and ra- diographs were retrospectively analyzed. Data collection included the patient's height, body weight (body mass index was calculated), tobacco use (smoking was defined as a daily consumption of 3 or more cigarettes), co-morbidity (hypertension and diabetes), and osteotomy size. Complications that required close observation without additional surgical treatment were defined as mild complications: 1) nondisplaced lateral cortex fracture, 2) neuropathy, 3) correction loss, 4) hematoma, 5) delayed union, 6) delayed wound healing, 7) postoperative stiffness, 8) hardware irritation, 9) tendinitis, and 10) hardware failure without associated symptoms. The other events that required additional surgical treatment were defined as major complications: 1) hardware failure with associated symptoms (severe pain and correction loss to preoperative alignment), 2) deep infection, and 3) nonunion. Patients without complications were classified into group 1 and those with complications into group 2.

To compare the radiologic and clinical outcomes between group 1 and group 2, we measured mechanical tibiofemoral angle, medial proximal tibial angle, and posterior slope of the tibia on the

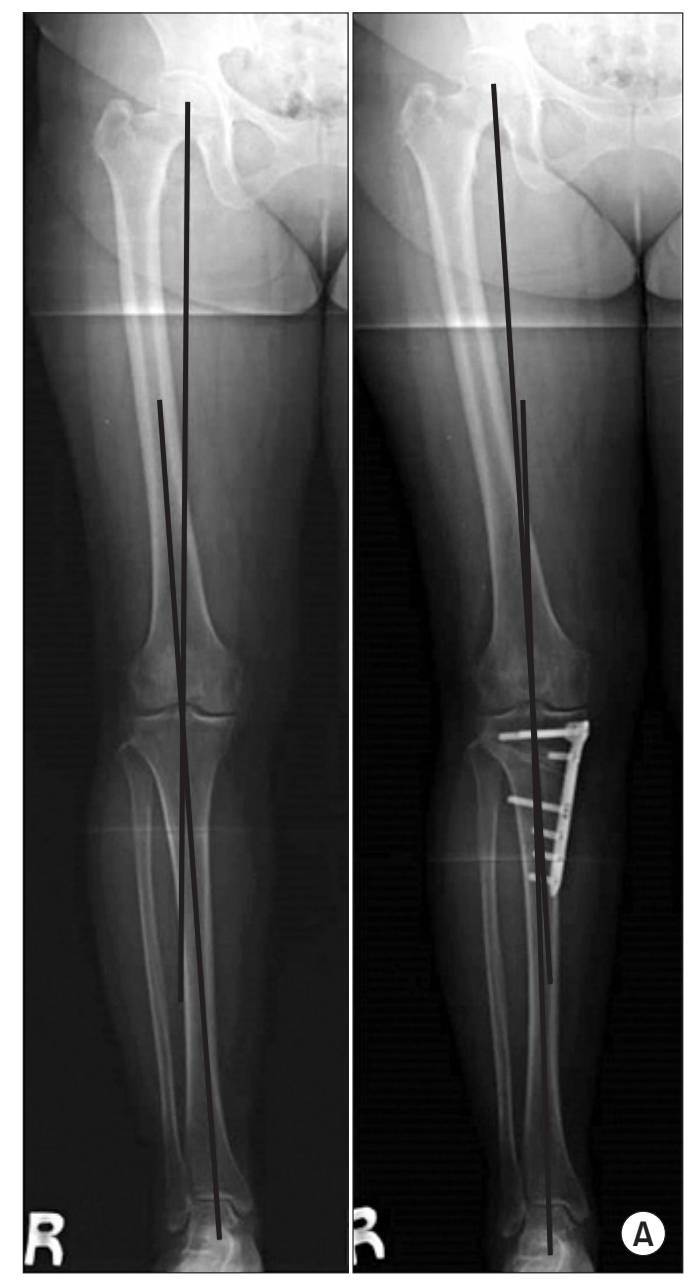

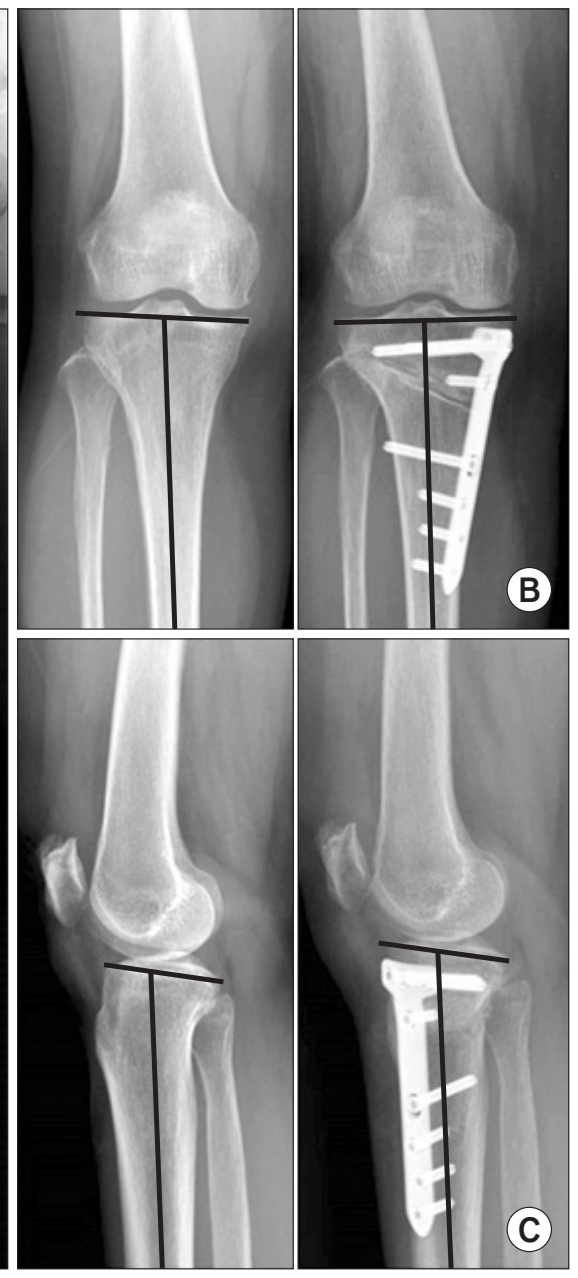

Fig. 1. Preoperative and postoperative 1 year radiographs. (A) Measurement of the mechanical tibiofemoral angle. (B) Measurement of the medial proximal tibia angle. (C) Measurement of the tibial posterior angle. 
preoperative and 1-year postoperative radiographs (Fig. 1). The Oxford Knee score and Knee Injury and Osteoarthritis Outcome score (KOOS) were also measured on the preoperative and 1-year postoperative radiographs.

In all patients, arthroscopic examination of medial compartment osteoarthritis was performed before MOWHTO. Depending on the condition of the knee joint, multiple drilling, meniscal repair, or meniscectomy was performed. After the arthroscopic procedure, an approximately $6-\mathrm{cm}$ longitudinal skin incision was made from just distal to the medial knee joint. The superficial medial collateral ligament and pes anserius were subperiosteally released from the tibia. Then, a radiolucent Hohmann retractor was inserted into the posterior surface of the proximal tibia to protect the neurovascular structures and expose the osteotomy site. Two Kirschner wires were inserted anteriorly and posteriorly from 3-4 cm distal to the medial knee joint and were directed at the "safe zone" between the circumference line of the fibula and the level of the tip of the fibular head under fluoroscopic guidance. Osteotomy was performed beneath the wire using an oscillating saw until the osteotomy line extends to $1 \mathrm{~cm}$ medial to the lateral tibial cortex. After a desired degree of correction was achieved, the locking plate was fixed and the defect for correction was grafted using an allograft. A long leg splint was applied after surgery. Isometric quadriceps exercise and straight leg-raising exercise were started. The long leg splint was removed at 1 week after surgery and continuous passive motion exercise was initiated. Partial weight bearing was performed until 6 postoperative weeks and full weight bearing was permitted at 6 weeks after surgery.

Statistical analyses were performed using MedCalc ver. 15.2.2

Table 1. Patient Demographics and Baseline Characteristics

\begin{tabular}{lc}
\hline \multicolumn{1}{c}{ Characteristic $(\mathrm{n}=167)$} & Mean \\
\hline Age $(\mathrm{yr})$ & $55.6 \pm 8.07$ \\
Sex $(\mathrm{M} / \mathrm{F})$, ratio & $0.34(43 / 124)$ \\
Operated side $(\mathrm{left} / \mathrm{right})$ & $97 / 70$ \\
Body mass index $\left(\mathrm{kg} / \mathrm{m}^{2}\right)$ & $25.42 \pm 3.42$ \\
Active smoker $(\%)$ & $13(7.8)$ \\
Comorbidity $(\%)$ & \\
Hypertension & $40(23.9)$ \\
Diabetes & $41(24.6)$ \\
Preop mTFA $\left(^{\circ}\right)$ & $186.59 \pm 3.52$ \\
Preop MPTA $\left(^{\circ}\right)$ & $86.40 \pm 1.62$ \\
Preop slope of tibia $\left(^{\circ}\right)$ & $11.90 \pm 3.42$ \\
\hline
\end{tabular}

Values are presented as mean \pm standard deviation or number.

Preop: preoperative, mTFA: mechanical tibiofemoral angle, MPTA: medial proximal tibial angle.
(MedCalc Software, Ostend, Belgium). Differences among groups were compared using the independent samples $t$-test and chi-square test. Odds ratios (OR) were calculated using multiple logistic regression analysis adjusted for the influences of several factors on complications. P-values less than 0.05 were considered to be statistically significant.

\section{Results}

Patient demographics and clinical characteristics of the patients are described in Table 1. Overall, complications were observed in 49 patients (29.3\%). Minor complications requiring observation or nonsurgical treatment included simple lateral cortex fracture (26 cases, 15.6\%), neuropathy (6 cases, 3.6\%), correction loss (4 cases, $2.4 \%$ ), hematoma (4 cases, $2.4 \%$ ), delayed union (4 cases, $2.4 \%$ ), delayed wound healing ( 4 cases, $2.4 \%$ ), postoperative stiffness ( 2 cases, $1.2 \%$ ), pain from skin irritation due to plate ( 2 cases, $1.2 \%$ ), tendinitis ( 2 cases, $1.2 \%$ ), and hardware failure without associated symptoms ( 1 case, $0.6 \%$ ). Major complications requiring additional surgical treatment included hardware failure with associated symptoms such as severe pain and correction loss (1 case, $0.6 \%$ ), deep infection ( 1 case, $0.6 \%$ ) and nonunion ( 1 case, $0.6 \%$ ) (Table 2).

Table 2. Distribution of Complications

\begin{tabular}{lcc}
\hline \multicolumn{1}{c}{ Characteristic } & Complication (\%) & No. of cases \\
\hline Minor complications & 15.6 & 26 \\
Lateral cortex fracture & 3.6 & 6 \\
Neuropathy & 2.4 & 4 \\
Correction loss (less than mTFA 180 ${ }^{\circ}$ ) & 2.4 & 4 \\
Hematoma & 2.4 & 4 \\
Delayed union & 2.4 & 4 \\
Delayed wound healing (superficial & & \\
$\quad$ infection) & 1.2 & 2 \\
Postoperative stiffness & 1.2 & 2 \\
Hardware irritation & 1.2 & 2 \\
Tendinitis & 0.6 & 1 \\
Hardware failure without associated & & 1 \\
$\quad$ symptoms & & 1 \\
Major complications & 0.6 & 1 \\
Hardware failure with associated & & \\
$\quad$ symptoms & 0.6 & \\
Deep infection & 0.6 & \\
Nonunion & & \\
Total & &
\end{tabular}

mTFA: mechanical tibiofemoral angle. 
The results of multiple logistic regression analysis adjusted for the occurrence of complications are shown in Table 3.

Preoperatively, there were no statistically significant differences regarding radiographic measurements between group 1 and group 2 (Fig. 2). At postoperative 1 year, there were no significant differences in radiologic measurements between group 1 and group 2 (Fig. 2). Preoperatively, there were no statistically significant differences regarding the knee scores between group 1 and group 2 (Fig. 3). Postoperatively, there was a significant difference in KOOS pain score between group 1 and group $2(\mathrm{p}=0.044)$, but there were no significant differences in the other knee scores (Fig. $4)$.

\section{Discussion}

In this study, we identified a $29.3 \%$ complication rate after MOWHTO for varus osteoarthritis of the knee. Almost all cases (94.8\%) were minor complications that were possible to recover without surgical management. There were few major complications requiring surgical management (only 3 cases). In addition, there were no popliteal artery damage, systemic complications, and cardiac or respiratory events. There were no significant differences in radiologic measurements between group 1 and group 2 at the first postoperative year. Even though there was a significant difference in the KOOS pain score between group 1 and group 2, there were no significant differences in clinical outcomes. Thus, we believe that complications of MOWHTO do not have significant influence on clinical outcomes.

In this study, we classified the 167 patients into two groups depending on the presence of complications and investigated the impact of complications on the radiologic and clinical outcomes. Minor complications requiring observation or nonsurgical treatment included simple lateral cortex fracture (26 cases, 15.6\%), neuropathy (6 cases, $3.6 \%$ ), correction loss ( 4 cases, $2.4 \%$ ), hematoma (4 cases, $2.4 \%$ ), delayed union ( 4 cases, $2.4 \%$ ), delayed wound healing ( 4 cases, $2.4 \%$ ), postoperative stiffness ( 2 cases, $1.2 \%)$, pain from skin irritation due to plate ( 2 cases, $1.2 \%$ ), tendinitis ( 2 cases, $1.2 \%$ ), and hardware failure without associated symptoms ( 1 case, $0.6 \%)$. Major complications requiring additional surgical treatment included hardware failure with associated symptoms (1 case, $0.6 \%$ ), deep infection (1 case, $0.6 \%$ ) and nonunion ( 1 case, $0.6 \%$ ). In previous studies, the incidence of complications after MOWHTO ranged from $1.9 \%$ to $55 \%^{3,8-12)}$. Complication rates of MOWHTO using the Puddu plate (Arthrex, Naples, FL, USA) have been reported to range from $30.7 \%$ to $45.8 \%^{9,12-15)}$. Although studies on complications of MOWHTO

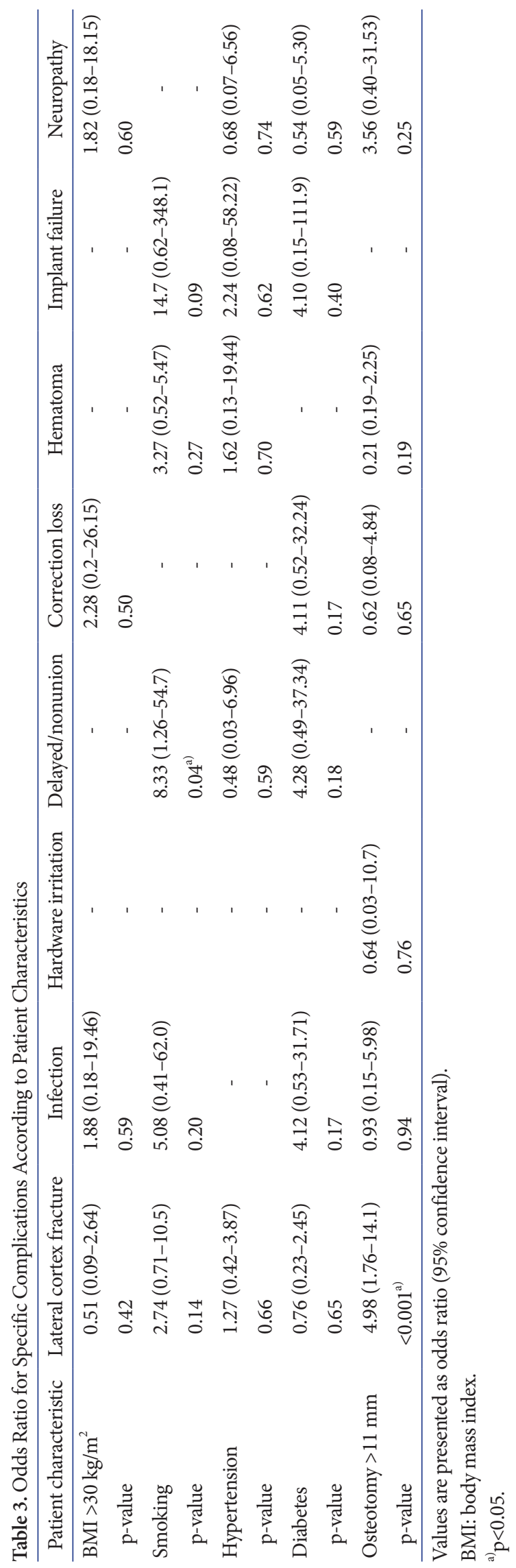




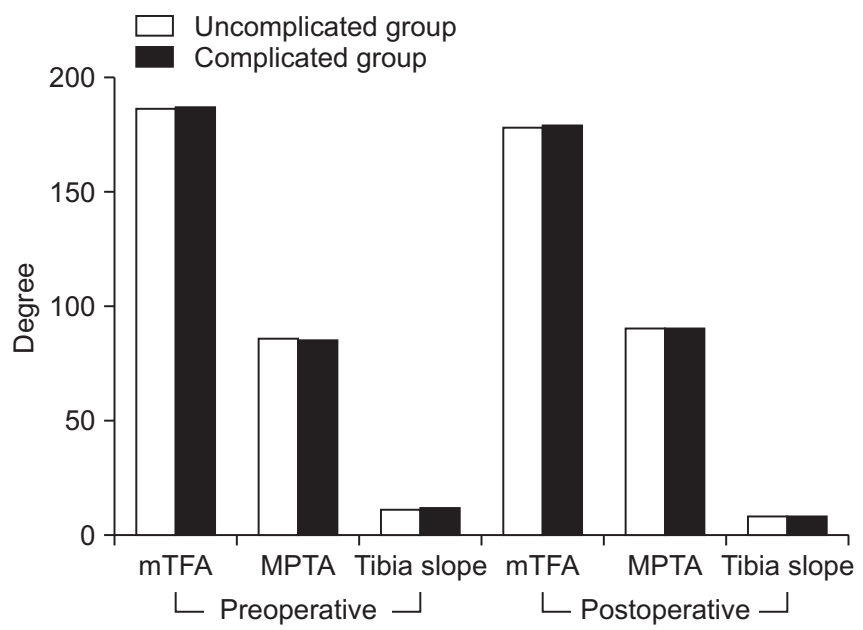

Fig. 2. There were no significant differences in radiologic measurements between group 1 and group 2 at preoperative and postoperative 1 year. mTFA: mechanical tibiofemoral angle, MPTA: medial proximal tibial angle.

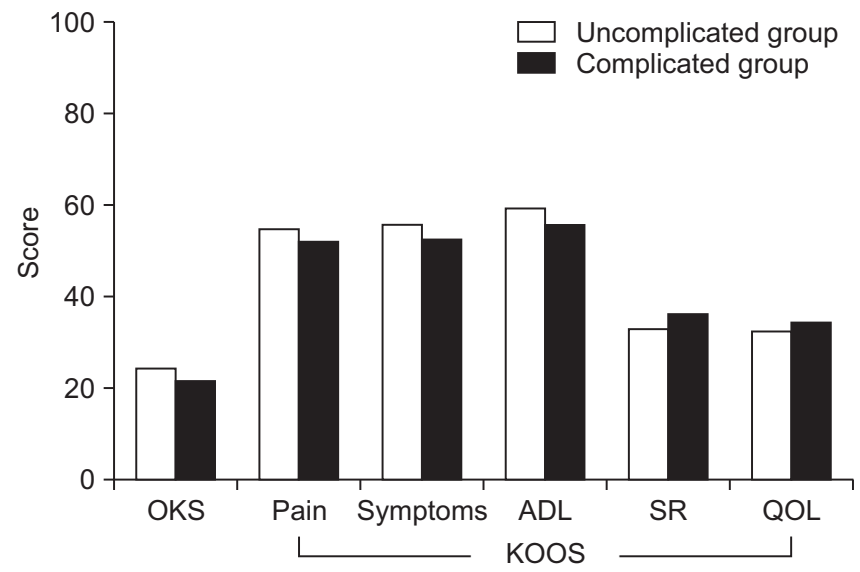

Fig. 3. There were no significant differences in preoperative clinical outcome scores between group 1 and group 2. OKS: Oxford Knee score, KOOS: Knee Injury and Osteoarthritis Outcome score, ADL: activities of daily living, SR: sports and recreation, QOL: quality of life.

using the TomoFix plate are relatively rare, it has been associated lower rates of complications than those using the Puddu plate (Fig. $5)^{3,7,11}$. Valkering et al. ${ }^{7)}$ reported only 4 cases $(10 \%)$ of superficial infection of 40 MOWHTOs and other complications were not observed. Niemeyer et al. ${ }^{3)}$ reported that the overall complication rate was 6 cases (8.6\%) including 1 case of intra-articular fracture, 3 cases of overcorrection, and 2 cases of delayed union. However, these studies on complications of MOWHTO using the TomoFix plate had a small number of subjects and reported complications that were not much related to the locking plate. The current study had a larger number of subjects (167 cases) than previous studies and thus could evaluate complications more precisely. Because of

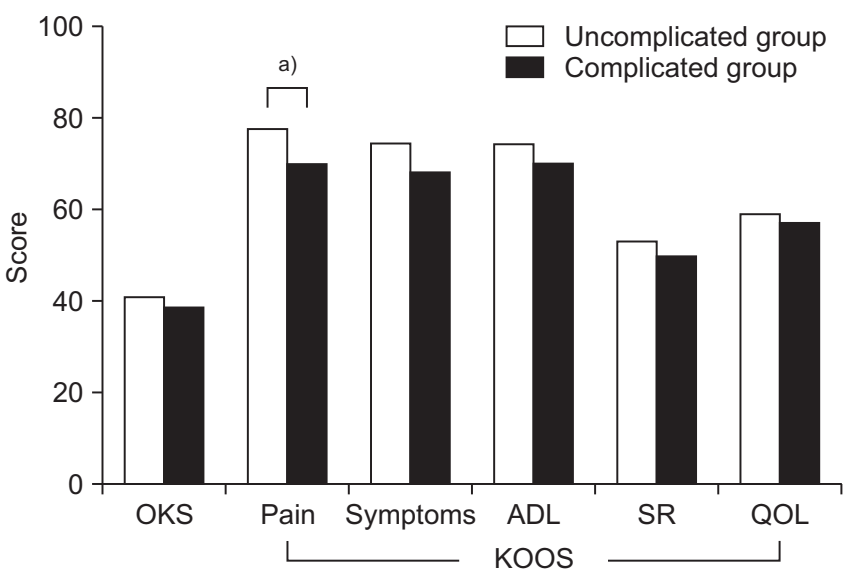

Fig. 4. Despite the KOOS (pain) showed significant difference between group 1 and group 2, the clinical outcome scores were similar in general between the two groups. OKS: Oxford Knee score, KOOS: Knee Injury and Osteoarthritis Outcome score, ADL: activities of daily living, SR: sports and

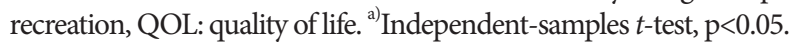

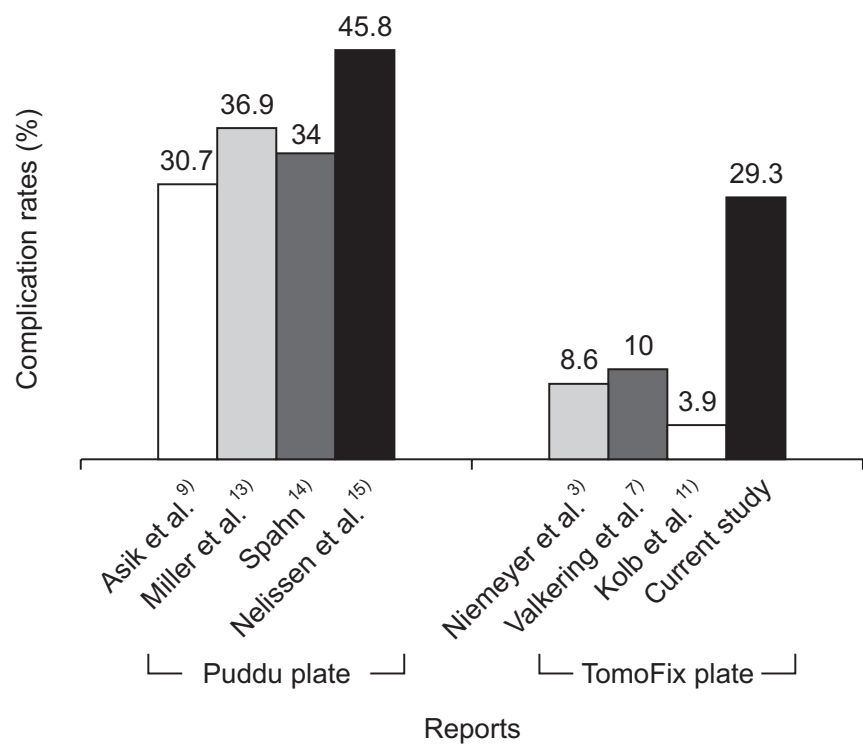

Fig. 5. The complication rates in our study are lower than those in studies using the Puddu plate but higher than those in studies using the TomoFix plate.

the inclusion of minor complications in the analysis, we observed higher complication rates in our patients than those in the other studies (Table 4$)^{3,7,9,13-16)}$.

In the present study, the most common complication was lateral cortex fracture that had the incidence of 26/167 (15.6\%). The incidence of the complication in the present study was similar to that reported by Esenkaya and Elmali ${ }^{12}(14 / 74,18.9 \%)$ and Nelissen et al. ${ }^{15)}(9 / 49,18.4 \%)$. Of total, 16/26 (61.5\%) could be identified during surgery and the other 10/26 (38.5\%) were diagnosed 


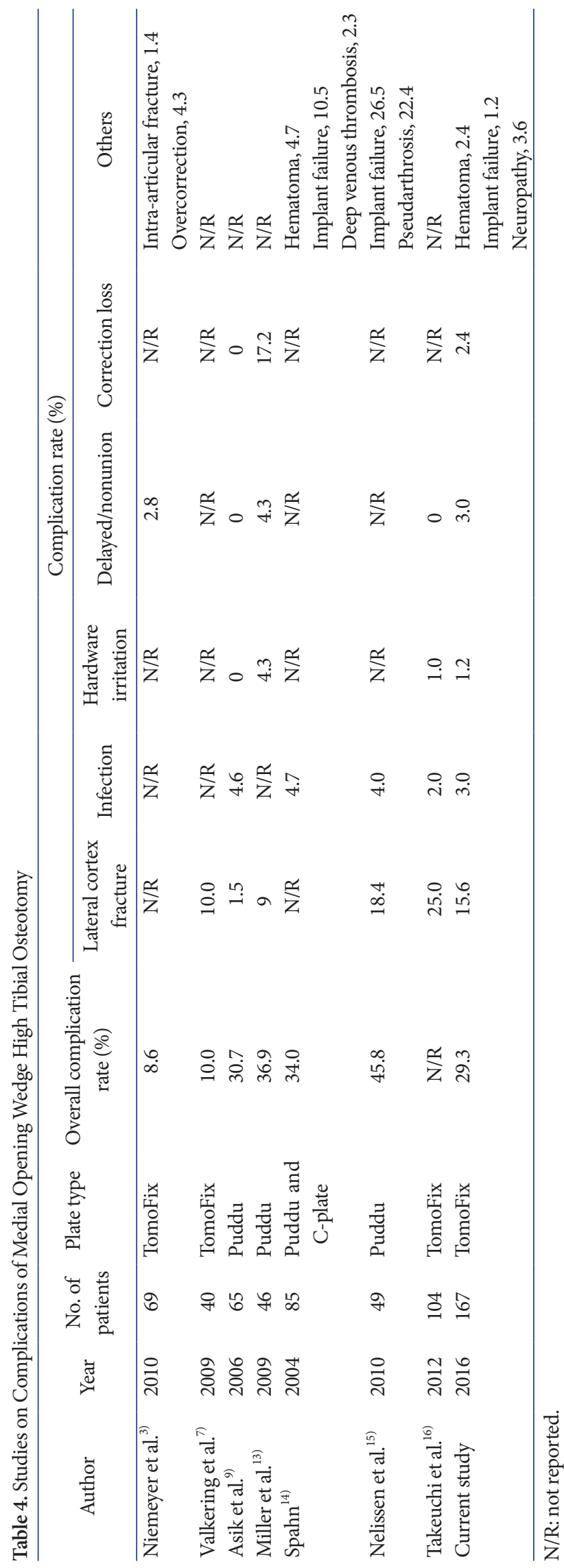

based on postoperative radiographs. Similarly, Miller et al. ${ }^{13)}$ identified $50 \%$ of complications in their patients after surgery. All lateral cortex fractures were nondisplaced lateral cortex fractures and there was no case of displaced lateral cortex fracture or tibial plateau fracture. In the study, OR were calculated to identify risk factors that could significantly influence the occurrence of complications (Table 3). The mean medial opening size in the lateral cortex fracture group and non-fracture group was $11.7 \pm 2.8 \mathrm{~mm}$ and $10.38 \pm 2.27 \mathrm{~mm}$, respectively and showed a significant difference between groups $(\mathrm{p}=0.025)$. Also, it was found that the medial opening size $(>11 \mathrm{~mm})$ was a significant risk factor $(\mathrm{OR}=4.98)$ of a lateral cortex fracture. So they suggested that when the medial opening size increases, more attention should be paid to prevent a lateral cortex fracture. Stoffel et al. ${ }^{17)}$ investigated the initial stability of the Puddu plate and TomoFix plate biomechanically and concluded that sufficient residual stability remained in the knees with the TomoFix plate and careful partial weight bearing was possible. So, patients with nondisplaced lateral cortex fractures received the same rehabilitation management. In the study, 25 (96.1\%) of 26 cases had bony consolidations without any problems at 1-year follow up and 1 case (3.9\%) had reoperation because of nonunion.

Definitions of delayed union vary depending on authors. In the present study, delayed union was defined as insufficient bone healing at 3 postoperative months and subsequently evident bone healing until 6 postoperative months. The incidence of delayed union of the present study was $4 / 167$ (2.4\%). Similar to present study, the incidences of delayed union after MOWHTO using the TomoFix plate reported in the literature are between $0 \%$ and $1.1 \%^{18,19)}$. All patients with delayed union had bony consolidations and the mean duration of consolidation was 11.3 months. The rate of nonunion was much lower ( 1 case, $0.6 \%$ ), which required reoperation. Staubli et al. ${ }^{19)}$ reported 2 patients with delayed union were heavy cigarette smokers. In several studies, smoking has been reported as a risk factor negatively influencing bone union irrespective of the osteotomy method used ${ }^{20,21}$. van Houten et al. $\left.{ }^{20}\right)$ reported smoking as a risk factor $(\mathrm{OR}=4.1)$ for bone healing. Our study also identified smoking as a relevant factor that significantly influenced delayed union and nonunion $(\mathrm{OR}=8.33)$.

After swelling of the operation site was reduced, 2 patients (1.2\%) complained of severe pain (numerical rating scale more than 5) related to direct local irritation of the TomoFix plate. In these patients, metal removal was carried out at postoperative 1 year, and the pain related to direct local irritation disappeared.

The incidences of hardware failure in the literature are between 


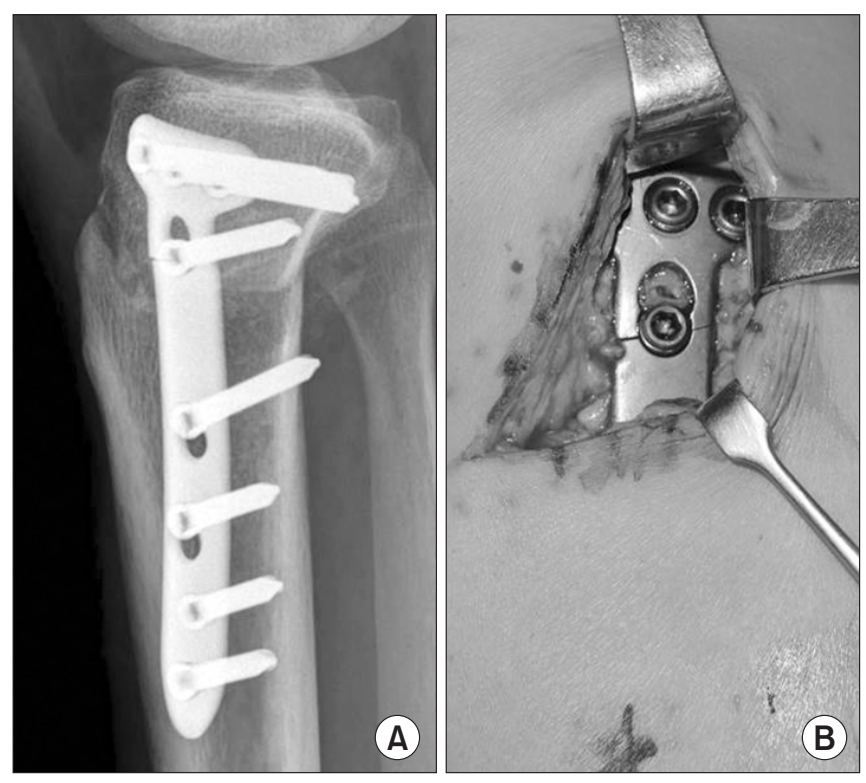

Fig. 6. Postoperative radiograph (A) and intraoperative photograph (B) in revision surgery showing locking plate failure at the osteotomy level.

$3.6 \%$ and $6.1 \%{ }^{14,15)}$; however, these studies investigated hardware failure after MOWHTO using a short spacer plate. Schroter et al. $^{22)}$ described that no hardware failure was observed in the knees with a locking plate. Floerkemeier et al. ${ }^{23)}$ reported 1 TomoFix hardware failure $(0.2 \%)$. In the present study, hardware failure was observed in 2 cases: 1 case was screw failure identified during operation for metal removal and the other case was accompanied by severe pain and correction loss. The patient who had a lateral cortex fracture carried out full weight bearing ambulation before 6 postoperative weeks and returned early to sports activities after surgery. He received reoperation for metal exchange and recorrection (Fig. 6).

At postoperative 1 year, there were no significant differences in radiologic measurements between group 1 and group 2. Postoperatively, radiologic measurement values did not change in group 2 except for 4 cases with correction loss. So, we could not find any statistically significant differences in radiologic measurements between the groups.

There was a significant difference in KOOS pain score between group 1 and group 2 ( $\mathrm{p}=0.044$ ) (Fig. 4); however, there were no significant differences in other knee scores. Almost all complications were recovered within postoperative 1 year. So, there were no significant differences in most patient-reported clinical knee scores. But, patients with skin irritation due to the locking plate, tendinitis, and major complication complained of severe pain at more than 1 year after surgery. Thus, a statistically significant difference was noted in the KOOS pain score between group 1 and group 2. Martin et al. ${ }^{6}$ reported that class 1 (no additional treatment required) and class 2 (short-term nonoperative treatment required) adverse events did not affect clinical outcomes. Patients with class 3 (additional surgery or long-term non-operative treatment required) adverse events had significantly lower total Western Ontario and McMaster Osteoarthritis Index scores at 6 postoperative months but not at 12 or 24 postoperative months. These results were similar to the clinical outcomes of the present study.

The present study has several limitations. First, it is the retrospective review of data. To limit bias, an orthopedic surgeon not involved in patient treatment evaluated the medical records, operative notes, and radiographs. Second, the follow-up period was relatively short; a longer follow-up may result in different clinical outcomes. So, long-term evaluation of complications after MOWHTO should be conducted. However, considering that the purpose of this study was to investigate complications, not to evaluate the overall outcome, we believed the 1 year follow-up would be sufficient.

\section{Conclusions}

MOWHTO using a locking plate can be a safe and reliable procedure for medial osteoarthritis of the knee. Our data showed that most complications were minor and recovered without any problems. Most complications did not have a significant impact on radiologic and clinical outcomes. Despite the low incidence of major complications, we suggest that more attention needs to be paid to decrease major complications.

\section{Conflict of Interest}

No potential conflict of interest relevant to this article was reported.

\section{References}

1. Lee DC, Byun SJ. High tibial osteotomy. Knee Surg Relat Res. 2012;24:61-9.

2. Chae DJ, Shetty GM, Wang KH, Montalban AS Jr, Kim JI, Nha KW. Early complications of medial opening wedge high tibial osteotomy using autologous tricortical iliac bone graft and T-plate fixation. Knee. 2011;18:278-84.

3. Niemeyer P, Schmal H, Hauschild O, von Heyden J, SJ, Sn NP, Kostler W. Open-wedge osteotomy using an internal plate fixator in patients with medial-compartment gonar- 
thritis and varus malalignment: 3-year results with regard to preoperative arthroscopic and radiographic findings. Arthroscopy. 2010;26:1607-16.

4. Frigg R. Locking Compression Plate (LCP): an osteosynthesis plate based on the Dynamic Compression Plate and the Point Contact Fixator (PC-Fix). Injury. 2001;32 Suppl 2:63-6.

5. Perren SM. Evolution of the internal fixation of long bone fractures: the scientific basis of biological internal fixation: choosing a new balance between stability and biology. J Bone Joint Surg Br. 2002;84:1093-110.

6. Martin R, Birmingham TB, Willits K, Litchfield R, Lebel ME, Giffin JR. Adverse event rates and classifications in medial opening wedge high tibial osteotomy. Am J Sports Med. 2014;42:1118-26.

7. Valkering KP, van den Bekerom MP, Kappelhoff FM, Albers GH. Complications after TomoFix medial opening wedge high tibial osteotomy. J Knee Surg. 2009;22:218-25.

8. van den Bekerom MP, Patt TW, Kleinhout MY, van der Vis HM, Albers GH. Early complications after high tibial osteotomy: a comparison of two techniques. J Knee Surg. 2008;21:68-74.

9. Asik M, Sen C, Kilic B, Goksan SB, Ciftci F, Taser OF. High tibial osteotomy with Puddu plate for the treatment of varus gonarthrosis. Knee Surg Sports Traumatol Arthrosc. 2006;14:948-54.

10. Amendola A, Fowler PJ, Litchfield R, Kirkley S, Clatworthy M. Opening wedge high tibial osteotomy using a novel technique: early results and complications. J Knee Surg. 2004;17:164-9.

11. Kolb W, Guhlmann H, Windisch C, Kolb K, Koller H, Gr, Gr H P. Opening-wedge high tibial osteotomy with a locked low-profile plate. J Bone Joint Surg Am. 2009;91:2581-8.

12. Esenkaya I, Elmali N. Proximal tibia medial open-wedge osteotomy using plates with wedges: early results in 58 cases. Knee Surg Sports Traumatol Arthrosc. 2006;14:955-61.

13. Miller BS, Downie B, McDonough EB, Wojtys EM. Complications after medial opening wedge high tibial osteotomy. Arthroscopy. 2009;25:639-46.

14. Spahn G. Complications in high tibial (medial opening wedge) osteotomy. Arch Orthop Trauma Surg. 2004;124:64953 .

15. Nelissen EM, van Langelaan EJ, Nelissen RG. Stability of medial opening wedge high tibial osteotomy: a failure analysis. Int Orthop. 2010;34:217-23.

16. Takeuchi R, Ishikawa $H$, Kumagai $K$, Yamaguchi $Y$, Chiba N, Akamatsu Y, Saito T. Fractures around the lateral cortical hinge after a medial opening-wedge high tibial osteotomy: a new classification of lateral hinge fracture. Arthroscopy. 2012;28:85-94.

17. Stoffel K, Stachowiak G, Kuster M. Open wedge high tibial osteotomy: biomechanical investigation of the modified Arthrex Osteotomy Plate (Puddu Plate) and the TomoFix Plate. Clin Biomech (Bristol, Avon). 2004;19:944-50.

18. Lobenhoffer P, Agneskirchner JD. Improvements in surgical technique of valgus high tibial osteotomy. Knee Surg Sports Traumatol Arthrosc. 2003;11:132-8.

19. Staubli AE, De Simoni C, Babst R, Lobenhoffer P. TomoFix: a new LCP-concept for open wedge osteotomy of the medial proximal tibia: early results in 92 cases. Injury. 2003;34 Suppl 2:B55-62.

20. van Houten AH, Heesterbeek PJ, van Heerwaarden RJ, van Tienen TG, Wymenga AB. Medial open wedge high tibial osteotomy: can delayed or nonunion be predicted? Clin Orthop Relat Res. 2014;472:1217-23.

21. Schroter S, Freude T, Kopp MM, Konstantinidis L, DL, Da S, Stockle U, van Heerwaarden R. Smoking and unstable hinge fractures cause delayed gap filling irrespective of early weight bearing after open wedge osteotomy. Arthroscopy. 2015;31:254-65.

22. Schroter S, Gonser CE, Konstantinidis L, Helwig P, Albrecht D. High complication rate after biplanar open wedge high tibial osteotomy stabilized with a new spacer plate (Position HTO plate) without bone substitute. Arthroscopy. 2011;27:644-52.

23. Floerkemeier S, Staubli AE, Schroeter S, Goldhahn S, Lobenhoffer P. Outcome after high tibial open-wedge osteotomy: a retrospective evaluation of 533 patients. Knee Surg Sports Traumatol Arthrosc. 2013;21:170-80. 